\section{UJMM

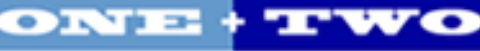

Volume 10 | 2020 Spring 2020

\section{Undergraduate Journal of Mathematical} Modeling: One + Two

2020

\title{
Optimization of Handicap Ramp
}

Tyler Schilling

University of South Florida

Advisors:

Arcadii Grinshpan, Mathematics and Statistics

Elijah Allen, Allen Concrete and Masonry, Naples, Florida

Problem Suggested By: Elijah Allen

Follow this and additional works at: https://digitalcommons.usf.edu/ujmm

Part of the Mathematics Commons

UJMM is an open access journal, free to authors and readers, and relies on your support:

Donate Now

\section{Recommended Citation}

Schilling, Tyler (2020) "Optimization of Handicap Ramp," Undergraduate Journal of Mathematical Modeling: One + Two: Vol. 10: Iss. 2, Article 6.

DOI: https://doi.org/10.5038/2326-3652.10.2.4919

Available at: https://digitalcommons.usf.edu/ujmm/vol10/iss2/6 


\title{
Optimization of Handicap Ramp
}

\begin{abstract}
The objective of this project is to minimize the cost of building a handicap ramp. This is done by introducing an equation that represents the total cost of the construction, including labor and materials. Variables are then defined in terms of block length $l$, allowing for an equation with one variable to be graphed and derived. This equation then undergoes the first derivative test to find a value of $I$ that would create a minimum output for cost. This value is then compared to the physical constraints of the project allowing for a realistic minimum cost to be found. The project concludes with finding the minimum total cost of $\$ 20,043$. To meet this minimum cost the number of stacks is 18 , the number of triangular prism blocks is 18, and the number of rectangular prism blocks is 171 .
\end{abstract}

\section{Keywords}

optimization, cubic equation, first derivative test, triangular and rectangular blocks

\section{Creative Commons License}

(c) (i) (3)

This work is licensed under a Creative Commons Attribution-Noncommercial-Share Alike 4.0 License. 


\section{PROBLEM STATEMENT}

A construction company needs to create a ramp that is handicap accessible to an entrance 5 meters off the ground. This will be done by stacking rectangular prism blocks of width 1 meter, length $l$, and height $h$ along with right triangular prism blocks of same base length $l$, same height $h$, and same width of 1 meter. Stacks will have a right triangular prism on top and a number of rectangular prism blocks starting with zero and increasing by one block per stack until the 5 meters height is reached. The cost per cubic meter of rectangular prism block material is $\$ 100$ and the cost per cubic meter of triangular prism block material is $\$ 500$. There is also a $\$ 10$ labor cost for each piece to be cut and placed into the ramp. By law handicap ramps must have a slope of $1 / 12$. What number of stacks, square blocks, and triangular blocks would minimize the cost of the ramp and what is this minimum cost?

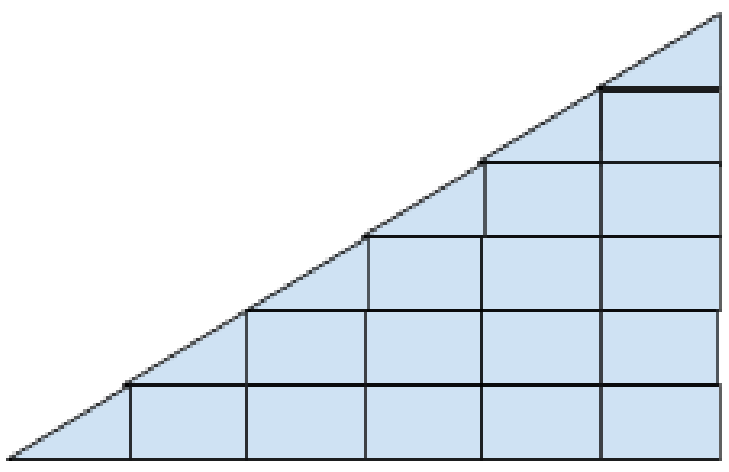

Figure 1: Blocks Forming Handicap Ramp 


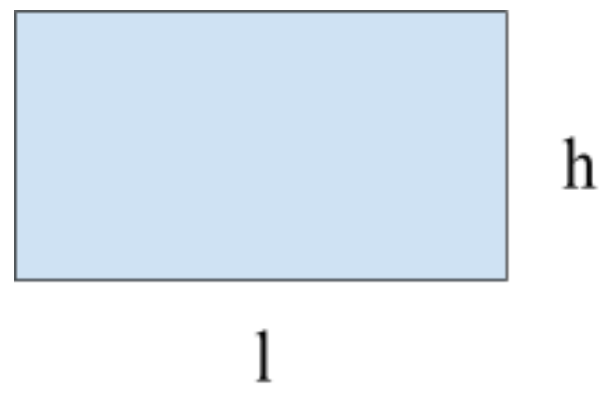

Figure 2: Labeled Rectangular Prism

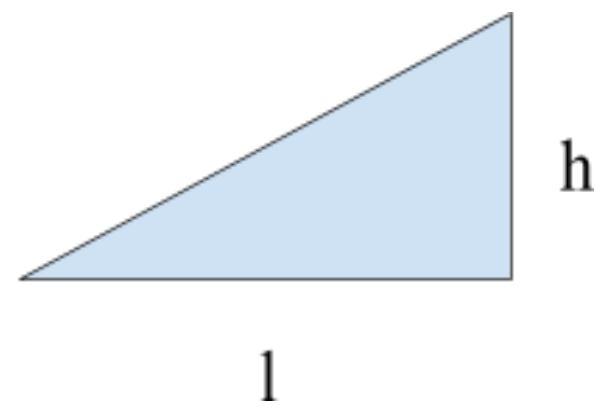

Figure 3: Labeled Triangular Prism

\section{MOTIVATION}

In engineering, minimization of cost is of the utmost importance, along with safety. Competition is very high, and offering a service at low cost is an extremely important factor. Construction of any structure must be done in a timely and cost efficient manner. Finding what the lowest cost is and more importantly how to achieve it and present your findings is what allows engineers to be able to do their work. The goal is not only finding the minimum cost of the project, but also determining how it will be done in terms of materials and labor.

\section{MATHEMATICAL DESCRIPTION AND SOLUTION APPROACH}

The quantity to be optimized in this situation is cost or $C$. We will use subsets of this to show cost of labor $C_{L}$ and cost of materials $C_{M}$. We then get the equation for total cost:

$$
C=C_{L}+C_{M}
$$


We can then break down this equation into two other equations, one for the cost of labor and one for the cost of materials. Labor cost will be found by multiplying the cost for labor of $\$ 10$ per block times the number of blocks in total denoted by $N$. The cost for materials will be found by multiplying the cost per volume of rectangular prism blocks $\$ 100 / \mathrm{m}^{3}$ by the volume of rectangular prism blocks, $V_{R}$, and adding the cost per volume of triangular prism blocks $\$ 500 / \mathrm{m}^{3}$ multiplied by the volume of triangular prism blocks $V_{T}$.

$$
\begin{gathered}
C_{L}=10 \mathrm{~N} \\
C_{M}=100 \mathrm{~V}_{R}+500 V_{T}
\end{gathered}
$$

To find the total number of blocks $N$, we must first find the length of our ramp. We know the height is 5 meters and the slope must be $1 / 12$ for all handicap ramps. This means the base must be 12 times the height making it 60 meters long. With the length of the ramp we can then find the number of stacks $n$ by dividing 60 meters by the length of each stack: $n=60 / l$, provided that $n$ is a whole number. Now that we have the number of stacks and since the stacks start at one block and go up by one for each stack after, we can use summation notation to denote the number of pieces in total. This summation can be simplified to an expression which we will use in our final cost equation:

$$
N=\sum_{k=1}^{n} k=\frac{n(n+1)}{2}, \text { where } n=60 / l
$$


We then substitute the new equation in place of $N$ for equation (2) and we have the total labor cost in terms of $l$ :

$$
C_{L}=5(60 / l)(60 / l+1)
$$

We now must find the volume of all rectangular prisms and all triangular prisms to plug into equation (3). This can be done by multiplying the number of each type of block by the volume of each type of block (Volume of one rectangular prism denoted by $V_{r}$ and volume of one triangular prism denoted by $V_{t}$ ). The number of triangular prisms would be equal to the total number of stacks or $60 / /$ since there is only one triangular prism per stack. The number of rectangular prisms would be equal to the total number of blocks found in equation (4) minus the number of triangles $60 / l$.

$$
\begin{gathered}
V_{T}=(60 / l) V_{t} \\
V_{R}=[(60 / l)(60 / l+1) / 2-(60 / l)] V_{r}=\frac{\left[\left(\frac{60}{l}\right)^{2}-\left(\frac{60}{1}\right)\right] \mathrm{Vr}}{2}
\end{gathered}
$$

Now all we have left is to replace the volumes with an expression in terms of $l$. The volume of the rectangular prisms will be the length of $l$ times the height $h$ times the width of $l$. The volume of the triangular prisms will be one half of the length $l$ times the height $h$ times the width of 1 . Plugging these values in for volume in equations (6) and (7) we get the following.

$$
V_{T}=(60 / l)(h l / 2)=30 h
$$




$$
V_{R}=\frac{\left[\left(\frac{60}{l}\right)^{2}-\left(\frac{60}{1}\right)\right] l h}{2}=1800(h / l)-30 h
$$

Since the slope is $1 / 12$ that means the height $h$ is going to be equal to the length $l$ divided by 12 . We can substitute this in for $h$ in the previous equations (8) and (9).

$$
\begin{gathered}
V_{T}=5(l / 2) \\
V_{R}=150-5(l / 2)
\end{gathered}
$$

We can now plug these values into equation (3) to get the total cost of materials.

$$
C_{M}=15,000+1,000 l
$$

We can then substitute equation (5) and equation (12) into equation (1) to get the total cost in terms of 1 .

$$
C(l)=5(60 / l)(60 / l+1)+15,000+1,000 l
$$

Equation (13) is our final equation used before optimization. We can now use this equation and the first derivative test to find the absolute minimum of cost in relation to $l$ (Another option is to graph the function and find minimum points; this graph can be found in the Appendix). After taking the derivative $C^{\prime}(l)$, setting it equal to 0

$$
C^{\prime}(l)=1000-\frac{(300 l+36000)}{l^{3}}=0,
$$

and solving the corresponding cubic equation

$$
10 x^{3}-3 x-360=0,
$$


we get a single positive output of $\approx 3.3322$ for $l$ which gives us the ordered pair:

(3.3322, 20,043.33). Indeed, equation $(15)$ has one real root $\approx 3.3322$ and two complex conjugate roots $\approx-1.666 \pm 2.833 i$.

The found output value is the minimum possible cost in terms of the equation however we must make sure it works in the context of the problem. To do this our value of the number of stacks must be a whole, positive number. In making equation (4) we determined that the number of stacks $n=(60 / l)$. When we plug in our solved value of 3.3322 we get a value of $n$ slightly over 18. By looking at the graph we can determine that the closest point that gives us a whole number of 18 for $n$ is $l=10 / 3$. This means that the point $(10 / 3,20,043.33)$ is our minimum point in the context of this problem.

\section{DISCUSSION}

In our problem statement we were interested in finding a minimum cost, number of stacks, square blocks, and triangular blocks. Using the information from our previous paragraph we can see the minimum cost is going to be $\$ 20,043.33$ and the number of stacks will be 18 . We know the number of triangular prism blocks is equal to the number of stacks at 18 and that the number of rectangular prism blocks can be found by plugging in $l$ of $10 / 3$ into equation (7) and removing the $V_{r}$. After doing this we find the number of rectangular prism blocks to be equal to 171, which constitutes the final answer we sought for.

\section{CONCLUSION AND RECOMMENDATIONS}

The goal of this project is to minimize the cost in building a handicap ramp. This is done by defining an equation for cost based on labor cost and material cost. These two costs are then 
expanded into equations in terms of $l$, i.e. the length of one block used in construction. After creating a final cost equation, the first derivative and graphing are used to find the minimum point of the equation. The data is then related to practical constraints of the numbers allowing us to find the lowest achievable cost of $\$ 20,043.33$. This can be done by using 18 stacks with 18 triangular prism blocks and 171 rectangular prism blocks in total. This data can now be presented in a concise manner to show the lowest possible cost for the project and how to achieve it.

For future projects of this type or even to build upon this project, I would find other companies or create other equations for possible costs that are greater or less for materials and labor and relate the minimum costs of the multiple equations. This could then be used to pick which company should be hired for construction. 


\section{NOMENCLATURE}

\begin{tabular}{|c|c|c|}
\hline Symbol & Description & Units \\
\hline$C$ & Cost & $\$$ \\
\hline$C_{L}$ & Labor Cost & $\$$ \\
\hline$C_{M}$ & Material Cost & $\$$ \\
\hline$V_{T}$ & $\begin{array}{c}\text { Volume of all Triangular } \\
\text { Prisms }\end{array}$ & $\mathrm{m}^{3}$ \\
\hline$V_{R}$ & $\begin{array}{c}\text { Volume of all Rectangular } \\
\text { Prisms }\end{array}$ & $\mathrm{m}^{3}$ \\
\hline$N$ & Total Number of Blocks & \# \\
\hline$l$ & Length & $\mathrm{m}$ \\
\hline$h$ & Height & $\mathrm{m}$ \\
\hline$V_{r}$ & $\begin{array}{c}\text { Volume of one Rectangular } \\
\text { Prism }\end{array}$ & $\mathrm{m}^{3}$ \\
\hline$V_{t}$ & $\begin{array}{c}\text { Volume of one Triangular } \\
\text { Prism }\end{array}$ & $\mathrm{m}^{3}$ \\
\hline$n$ & Number of Stacks & \# \\
\hline
\end{tabular}

\section{REFERENCES}

Stewart, James. Calculus: Early Transcendentals. Cengage Learning, 2016 


\section{APPENDIX}

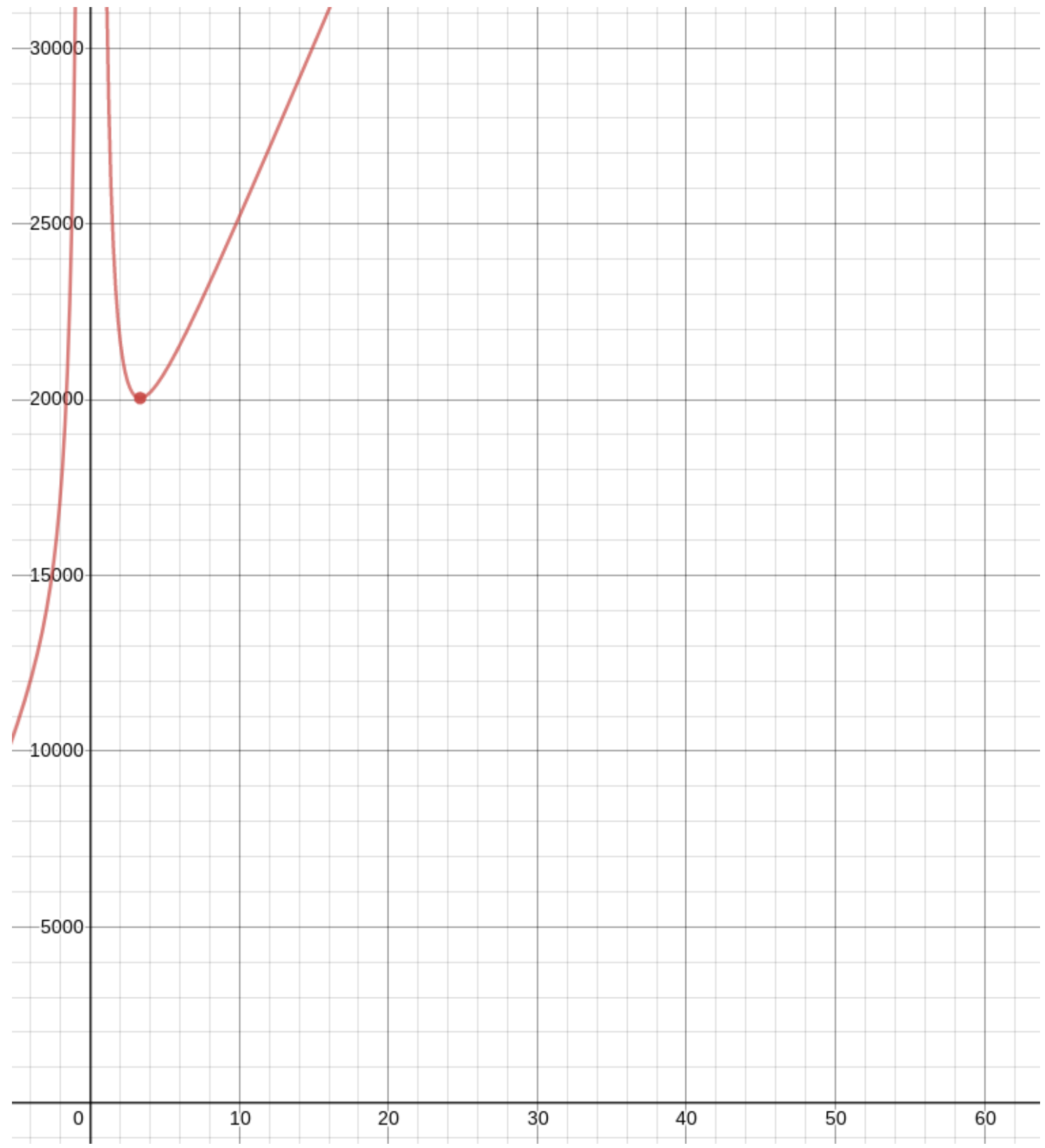

Figure 4: Graph of Cost Function $C=5(60 / l)(60 / l+1)+15,000+1,000 l$ 